\title{
Where is the neutral atomic gas in Hickson groups?^
}

\author{
L. Verdes-Montenegro ${ }^{1}$, M. S. Yun ${ }^{2}$, B. A. Williams ${ }^{3}$, W. K. Huchtmeier ${ }^{4}$, A. Del Olmo ${ }^{5}$, and J. Perea ${ }^{6}$ \\ 1 Instituto de Astrofísica de Andalucía, CSIC, Apdo. Correos 3004, 18080 Granada, Spain \\ 2 Astronomy Department, University of Massachusetts, Amherst, MA 01003, USA \\ e-mail: myun@astro.umass.edu \\ 3 University of Delaware, Newark, Delaware, USA \\ e-mail: baw@udel.edu \\ 4 Max-Planck-Institut für Radioastronomie Auf dem Hügel 69, 53121 Bonn, Germany \\ e-mail: huchtmeier@mpifr-bonn.mpg.de \\ 5 Instituto de Astrofísica de Andalucía, CSIC Apdo. Correos 3004, 18080 Granada, Spain \\ e-mail: chony@iaa.es \\ 6 Instituto de Astrofísica de Andalucía, CSIC Apdo. Correos 3004, 18080 Granada, Spain \\ e-mail: jaime@iaa.es
}

Received 7 May 2001 / Accepted 8 August 2001

\begin{abstract}
We have analyzed the total HI contents of 72 Hickson compact groups of galaxies (HCGs) and the detailed spatial distributions and kinematics of HI within a subset of 16 groups using the high angular resolution observations obtained with the VLA in order to investigate a possible evolutionary scenario for these densest systems in the present day galaxy hierarchy. For the more homogeneous subsample of 48 groups, we found a mean HI deficiency of $D e f_{\mathrm{HI}}=0.40 \pm 0.07$, which corresponds to $40 \%$ of the expected HI for the optical luminosities and morphological types of the member galaxies. The individual galaxies show larger degrees of deficiency than the groups globally, $D e f_{\mathrm{HI}}=0.62 \pm 0.09$ (24\% of the expected HI), due in most cases to efficient gas stripping from individual galaxies into the group environment visible in the VLA maps. The degree of deficiency is found to be similar to the central galaxies of Virgo and Coma cluster, and Coma I group, in spite of the significantly different characteristics (number of galaxies, velocity dispersion) of these environments. It does not seem plausible that a significant amount of extended HI has been missed by the observations. Hence phase transformation of the atomic gas should explain the HI deficiency. The groups richer in early type galaxies or more compact with larger velocity dispersions show a weak tendency to be more HI deficient. The detection rate of HCGs at X-ray wavelengths is larger for HI deficient groups, although the hot gas distribution and hence its origin is only known for a few cases. In the evolutionary scenario we propose, the amount of detected HI would decrease further with evolution, by continuous tidal stripping and/or heating. The $\mathrm{H}_{2}$ content also tends to be lower than expected for the galaxies in HI deficient groups, this may suggest that the HI stripping by frequent tidal interaction breaks the balance between the disruption of molecular clouds by star formation and the replenishment from the ambient HI.
\end{abstract}

Key words. galaxies: interactions - galaxies: kinematics and dynamics - galaxies: evolution - galaxies: structure galaxies: ISM - radio lines: galaxies

\section{Introduction}

The Hickson Compact Groups (Hickson 1982: HCGs) are characterized by a small number of members (4 to 10) with

\footnotetext{
Send offprint requests to: L. Verdes-Montenegro, e-mail: lourdes@iaa.es

* This work is partially based on observations made with the VLA operated by the National Radio Astronomy Observatory, a facility of the National Science Foundation operated under cooperative agreement by Associated Universities, Inc., ALFOSC, which is owned by the Instituto de Astrofísica de Andalucía (IAA, CSIC) and operated at the Nordic Optical Telescope (NOT) under agreement between IAA and the NBIfA of the Astronomical Observatory of Copenhagen, and $1.5 \mathrm{~m}$ telescope of the Observatorio de Sierra Nevada, Granada, Spain, which is operated by the IAA (CSIC).
}

a low velocity dispersion $(<\sigma\rangle=200 \mathrm{~km} \mathrm{~s}^{-1}$, Hickson et al. 1992). Their projected galaxy densities are extremely high, similar to the cores of dense clusters, but they are found in low galaxy density environments, as a consequence of the isolation criterion used for their selection (Hickson 1982; Sulentic 1987). This combination of high galaxy density in the low density environment makes them unique and interesting laboratories for studying galaxy interaction and evolution.

The origin and the existence of Hickson compact groups constitute a matter of great interest and debate. Their short crossing times argue for short lifetimes, yet few merger candidates or postcursors are found (Zepf et al. 1991; Moles et al. 1994; Sulentic \& Rabaça 1994). Diaferio et al. (1994) proposed that compact groups could form 
continually out of rich groups. Governato et al. (1996) suggest that primordial merger events with ongoing acquisition of intruders might prevent the groups from merging entirely. These models seem at work in HCG 92 (Moles et al. 1997). On the other hand, well isolated groups with no obvious intruders also exist (e.g. HCG 96 VerdesMontenegro et al. 1997). Athanassoula et al. (1997) have shown that compact groups with an appropriate arrangement of luminous and dark matter can persist over several hundred million years, in agreement with the analysis of the dynamics of satellite galaxies (Perea et al. 2000).

The characteristics of the interstellar medium in HCGs are also not well understood. Elevated star formation activity induced by tidal interactions and resulting enhancement in FIR and CO emission are expected but not supported by observations (see Verdes-Montenegro et al. 1998 and references therein). The highly perturbed distributions of the molecular gas in the most $\mathrm{CO}$ deficient groups suggest that strong disruptions of gaseous disks and gas stripping by continuous tidal disruption may suppress star formation (Yun et al. 1997).

A detailed analysis of the morphology and the strength of interactions among the HCG galaxies is needed in order to better understand the way these compact groups form and evolve. Studies of neutral hydrogen (HI) can offer valuable insights into the dynamics of galaxy interaction and evolution (e.g. Yun et al. 1994; Hibbard \& van Gorkom 1997), and we were motivated to undertake a survey of HI distribution and content for a large sample of HCGs (Williams et al. 1997, 1999; Verdes-Montenegro et al. 2000a,b; Huchtmeier et al. 2000) in order to analyze the type and effect of the interactions that are taking place. In this paper we report the analysis of the HI content for 72 HCGs and combine this with the analysis of the high spatial and spectral resolution VLA HI observations for a subset of 16 groups. Detailed studies of the individual groups will be presented elsewhere (in preparation). We have determined the frequency of perturbed groups from the HI content, the morphology, and the nature of the anomalies in the HI distribution. A global HI deficiency in HCGs has been previously reported in the literature (Williams \& Rood 1987; Huchtmeier 1997). We explore here a large set of parameters that might account for the HI anomaly. The high spatial and kinematical resolution of the HI data should help restrict the boundary conditions used in hydrodynamical modeling of galaxy interactions.

\section{The Sample selection and the HI data}

\subsection{Single-dish sample}

We have investigated published data for all groups from the Hickson catalog (Hickson 1982) containing at least 3 concordant members (hence HCG 9, 11, 36, 41 and 77 have been excluded; Hickson et al. 1992). HCG 70 is a projected superposition of a triplet and a quadruplet at different redshifts. Hence there are a total of 96 true groups in the Hickson Catalog. The HI single-dish spectra are available for 72 groups, either from the Arecibo and Green Bank radiotelescopes (Williams \& Rood 1987) and/or from the $100 \mathrm{~m}$ Effelsberg antenna (Huchtmeier 1997), and a total of 58 groups are detected. There is good agreement in the fluxes of the 31 groups with duplicate measures, as indicated in Huchtmeier (1997). Williams \& Rood (1987) find that, on average, HCGs are 50\% deficient in HI compared to a sample of loose groups. Huchtmeier (1997) finds a large scattering in the ratios of integrated HI mass to optical luminosity, including strong HI deficiencies for a large number of objects.

\subsection{The VLA sample}

We have mapped a total of 16 HCGs using the VLA. Detailed analysis of six spiral dominated groups have already been published (HCG 18, Williams \& Van Gorkom 1988; HCG 31, 44 and 79, Williams et al. 1991; HCG 23 and 26, Williams \& Van Gorkom 1995), and the preliminary analysis for the 5 other spiral dominated groups have also been reported (Williams et al. 1997, 1999; VerdesMontenegro et al. 2000a,b). HCG 92 (Stephan's Quintet) was also previously studied at WSRT (Shostak et al. 1984). We recently obtained the VLA data for five new groups (HCG 40, 49, 54, 95 and 96) in an effort to expand the sample to include more representatives of different interaction scenarios found in HCGs. The new additions include the densest compact groups, where the strongest interactions are expected, as well as some groups that are known to be HI deficient, as HCG 40, 44 and 92. A summary of the parameters for all observations is given in Table 1. Single-dish fluxes are compared to the integrated fluxes for the 16 groups observed with the VLA (corrected for primary beam attenuation, and removing the contributions from neighbor galaxies detected in $\mathrm{HI}$ within the VLA beam but external to the single-dish beam). Only in two cases (HCG 33 ad HCG 95) are we concerned that the interferometer may have missed flux (about 50\%). On the other hand the VLA flux for HCG 16 is larger than the measured value with a single dish, due to an $\mathrm{HI}$ extent larger than the single-dish beam.

HCG 22, 26, 42, 48, 63, 65 and 91 have been observed with the ATNF interferometer, but maps are only published for HCG 22 and 26 (Price et al. 2000). The sensitivity of the ATNF observations of HCG 26 is considerably lower than for the VLA data. No information on the total recovered flux is given for HCG 22, so it is not possible to evaluate how representative the VLA flux distribution is of the total HI emission.

\section{HI content of the HCGs}

\subsection{The single-dish sample}

The atomic hydrogen mass of the 72 groups with singledish data is computed as $M(\mathrm{HI})=2.36 \times 10^{5} D^{2} S \Delta V$, where $D$ is the luminosity distance in Mpc (assuming $H_{0}=75 \mathrm{~km} \mathrm{~s}^{-1} \mathrm{Mpc}^{-1}$ ) and $S \Delta V$ is the velocity 
Table 1. Observational parameters of the interferometric observations.

\begin{tabular}{lcccccc}
\hline HCG & $\begin{array}{c}\text { VLA } \\
\text { configuration }\end{array}$ & \multicolumn{2}{c}{ Beam size } & $\begin{array}{c}\text { Channel } \\
\text { width }\end{array}$ & $\sigma\left(S_{\nu}\right)$ & $\sigma\left(N_{\mathrm{HI}}\right)^{a}$ \\
& & $\left({ }^{\prime \prime} \times{ }^{\prime \prime}\right)$ & $(\mathrm{kpc} \times \mathrm{kpc})$ & $\left(\mathrm{km} \mathrm{s}^{-1}\right)$ & $(\mathrm{mJy} / \mathrm{beam})$ & $\left(10^{18} \mathrm{~cm}^{-2}\right)$ \\
\hline 2 & $\mathrm{D}$ & $68 \times 58$ & $18.0 \times 16.2$ & 10.6 & 0.64 & 1.9 \\
16 & $\mathrm{C}+\mathrm{D}$ & $25 \times 19$ & $6.3 \times 5.0$ & 21.3 & 0.31 & 15.0 \\
18 & $\mathrm{C}$ & $20 \times 20$ & $5.2 \times 5.2$ & 21.2 & 0.50 & 28.6 \\
23 & $\mathrm{C}$ & $21 \times 17$ & $5.3 \times 6.6$ & 10.6 & 1.70 & 54.5 \\
26 & $\mathrm{C}$ & $25 \times 18$ & $15.3 \times 11.0$ & 21.8 & 0.40 & 20.9 \\
31 & $\mathrm{CnD}$ & $21 \times 18$ & $5.6 \times 4.8$ & 21.0 & 0.22 & 13.2 \\
& $\mathrm{CnB}$ & $16 \times 14$ & $4.2 \times 3.9$ & 10.6 & 1.50 & 76.6 \\
33 & $\mathrm{C}$ & $18 \times 16$ & $9.0 \times 8.3$ & 21.8 & 0.65 & 53.1 \\
40 & $\mathrm{C}$ & $23 \times 18$ & $10.1 \times 7.9$ & 10.8 & 0.65 & 18.3 \\
44 & $\mathrm{D}$ & $61 \times 60$ & $5.4 \times 5.4$ & 42.0 & 0.40 & 5.0 \\
49 & $\mathrm{C}$ & $41 \times 23$ & $26.3 \times 14.8$ & 11.0 & 0.51 & 6.4 \\
54 & $\mathrm{C}$ & $20 \times 16$ & $1.9 \times 1.5$ & 10.4 & 0.33 & 11.6 \\
79 & $\mathrm{CnD}$ & $22 \times 17$ & $7.1 \times 4.5$ & 21.0 & 0.86 & 52.1 \\
& $\mathrm{C}$ & $25 \times 16$ & $7.0 \times 4.5$ & 10.6 & 0.39 & 11.2 \\
88 & $\mathrm{C}$ & $24 \times 17$ & $9.2 \times 6.8$ & 21.4 & 0.43 & 24.3 \\
92 & $\mathrm{C}+\mathrm{Cs}+\mathrm{D}$ & $20 \times 19$ & $7.8 \times 7.3$ & 21.5 & 0.54 & 33.0 \\
95 & $\mathrm{C}+\mathrm{D}$ & $23 \times 16$ & $17.7 \times 12.4$ & 22.2 & 0.22 & 14.3 \\
96 & $\mathrm{C}+\mathrm{D}$ & $33 \times 25$ & $18.7 \times 14.3$ & 21.9 & 0.34 & 9.7 \\
\hline & & & & &
\end{tabular}

a Column density obtained integrating for a channelwidth.

integrated HI flux in $\mathrm{Jy} \mathrm{km} \mathrm{s}^{-1}$. Fluxes are taken from Williams \& Rood (1987) and Huchtmeier (1997). For each galaxy the predicted mass is calculated as a function of its optical luminosity and morphological type (taken from Hickson et al. 1989) via the relationships obtained by Haynes \& Giovanelli (1984) for a sample of 324 isolated galaxies. In fact 34 galaxies were excluded from their analysis since they were not detected. Those correspond mostly to early types, and for this reason larger errors can be expected in the relationships. This effect is considered in Sect. 4. The HI mass expected for each group is obtained as the sum of the calculated masses for all its spiral and lenticular members.

The HI deficiency is defined as Def $f_{\mathrm{HI}}=$ $\log \left[M(\mathrm{HI})_{\text {pred }}\right]-\log \left[M(\mathrm{HI})_{\text {obs }}\right]$. We also adopt the definition of the standard estimate of the error (s.e.e.) in the HI masses predicted from the optical luminosity $L_{\mathrm{B}}$ as defined by Haynes \& Giovanelli (1984). This is on the order of $\log [M(\mathrm{HI})]=0.20$ for the HCGs studied. We consider the HI content of a galaxy/group anomalous when its HI content deviates from the predicted value by more than twice the mean s.e.e. The expected HI masses for the groups were derived as sums of the expected HI masses for the individual member galaxies. This approach is used because it takes into account the morphological dependency of $M(\mathrm{HI})$ and the well known non-linearity in the $M(\mathrm{HI})-L_{\mathrm{B}}$ relation $\left(M(\mathrm{HI}) \sim L_{\mathrm{B}}^{0.63-0.84}\right.$; Haynes \& Giovanelli 1984). Finally it would imply in many cases to extrapolate the $M(\mathrm{HI})$ versus $L_{\mathrm{B}}$ relationships above the range of values used in their determination.

The observed and predicted HI masses are compared in Fig. 1 (the diagonal line indicates the locus of galaxies with normal HI content) and are listed in Table 2 along with the s.e.e. An histogram of the HI deficiency for the 72 groups, shown in the inset of Fig. 1, gives a mean value of $D e f_{\mathrm{HI}}=0.36 \pm 0.06$. The upper and lower limits have been taken into account by means of survival analysis. This value will be revised in Sect. 4 considering effects that might increase/decrease the HI deficiency.

\subsection{Groups mapped with the VLA}

In Fig. 2 the total observed masses of the 16 compact groups imaged with the VLA are compared with the expected one. The mean deficiency of the groups of the VLA subsample is $D e f_{\mathrm{HI}}=0.16 \pm 0.10$ even though it is biased towards the groups with the strongest HI detections. The largest deficiency, De $f_{\mathrm{HI}}=0.97$, is found in HCG 40, whose detected HI mass is only about $10 \%$ of the expected mass. HI excess is inferred for HCG 33, but this group consists mostly of ellipticals, and the optical luminosity (thus predicted HI mass) of the only spiral member H33c is highly uncertain due to a bright foreground star and large Galactic extinction $\left(b=-12.6^{\circ}\right)$. Only lower limits 
Table 2. HI masses for groups.

\begin{tabular}{|c|c|c|c|c|c|}
\hline $\mathrm{HCG}$ & $\begin{array}{r}\text { Distance } \\
(\mathrm{Mpc})\end{array}$ & $\begin{array}{l}\log \left[M(\mathrm{HI})_{\text {obs }}\right] \\
\quad\left(\log \left[M_{\odot}\right]\right)\end{array}$ & $\begin{array}{c}\log \left[M(\mathrm{HI})_{\text {pred }}\right]^{a} \\
\left(\log \left[M_{\odot}\right]\right)\end{array}$ & $\begin{array}{c}\text { s.e.e. } \\
\left(\log \left[M_{\odot}\right]\right)\end{array}$ & Remarks \\
\hline 1 & 136.6 & 10.53 & 10.42 & 0.19 & \\
\hline 2 & 57.8 & 10.33 & 10.33 & 0.19 & triplet \\
\hline 3 & 102.6 & 10.18 & 9.99 & 0.19 & triplet \\
\hline 4 & 112.7 & 10.31 & 10.34 & 0.20 & \\
\hline 5 & 165.5 & 9.77 & 10.05 & 0.32 & triplet \\
\hline 6 & 152.9 & 9.69 & 10.02 & 0.22 & \\
\hline 7 & 56.6 & 9.68 & 10.37 & 0.17 & \\
\hline 8 & 220.7 & $<10.09$ & 10.13 & 0.21 & no spiral members \\
\hline 10 & 64.6 & 10.10 & 10.34 & 0.21 & \\
\hline 14 & 73.5 & 9.61 & 9.71 & 0.27 & triplet \\
\hline 15 & 91.7 & 9.41 & 10.03 & 0.22 & \\
\hline 16 & 52.9 & $>10.42$ & 10.57 & 0.15 & \\
\hline 18 & 54.1 & 10.03 & 9.89 & 0.18 & false group \\
\hline 19 & 57.4 & 9.31 & 9.89 & 0.22 & triplet \\
\hline 20 & 195.8 & $<10.06$ & 9.41 & 0.28 & \\
\hline 21 & 101.0 & 10.36 & 10.25 & 0.21 & \\
\hline 22 & 36.1 & 9.13 & 9.68 & 0.24 & triplet \\
\hline 23 & 64.6 & 10.03 & 10.00 & 0.17 & \\
\hline 24 & 122.8 & $<9.54$ & 9.97 & 0.24 & \\
\hline 25 & 85.2 & 9.90 & 10.16 & 0.19 & \\
\hline 26 & 127.3 & 10.42 & 10.23 & 0.16 & \\
\hline 30 & 61.8 & 8.62 & 10.18 & 0.22 & \\
\hline 31 & 54.5 & 10.35 & 10.53 & 0.19 & \\
\hline 33 & 104.6 & 10.23 & 9.48 & 0.30 & \\
\hline 34 & 123.6 & 10.70 & 9.93 & 0.20 & \\
\hline 35 & 219.5 & $<10.12$ & 10.01 & 0.22 & \\
\hline 37 & 89.6 & 9.19 & 10.07 & 0.20 & \\
\hline 38 & 117.6 & 9.69 & 10.44 & 0.18 & triplet \\
\hline 40 & 89.6 & 9.14 & 10.11 & 0.18 & \\
\hline 42 & 53.3 & 9.40 & 9.18 & 0.36 & no spiral members \\
\hline 43 & 133.0 & 10.11 & 10.37 & 0.18 & \\
\hline 44 & 18.4 & 9.23 & 9.92 & 0.19 & \\
\hline 46 & 108.6 & $<9.22$ & 9.37 & 0.25 & no spiral members \\
\hline 47 & 127.7 & 9.93 & 10.21 & 0.21 & \\
\hline 48 & 37.7 & 8.52 & 9.34 & 0.22 & \\
\hline 49 & 133.8 & 10.54 & 10.16 & 0.19 & \\
\hline 51 & 103.8 & $<9.66$ & 10.17 & 0.17 & \\
\hline 53 & 82.8 & 10.04 & 10.42 & 0.21 & triplet \\
\hline 54 & 19.6 & 8.75 & 9.24 & 0.23 & false group \\
\hline 56 & 108.6 & 9.36 & 10.09 & 0.16 & \\
\hline 57 & 122.4 & 9.71 & 10.57 & 0.16 & \\
\hline 58 & 83.2 & 9.83 & 10.40 & 0.19 & \\
\hline 59 & 54.1 & 9.49 & 9.45 & 0.22 & no spiral members \\
\hline 61 & 52.1 & 9.96 & 10.04 & 0.20 & triplet \\
\hline 62 & 54.9 & $<9.06$ & 9.52 & 0.26 & no spiral members \\
\hline 64 & 145.2 & $<10.06$ & 10.29 & 0.19 & triplet \\
\hline 67 & 98.5 & 10.03 & 10.30 & 0.18 & \\
\hline 68 & 32.0 & 9.62 & 10.10 & 0.22 & \\
\hline 69 & 118.4 & 10.27 & 10.18 & 0.17 & \\
\hline
\end{tabular}


Table 2. continued.

\begin{tabular}{|c|c|c|c|c|c|}
\hline $\mathrm{HCG}$ & $\begin{array}{r}\text { Distance } \\
(\mathrm{Mpc}) \\
\end{array}$ & $\begin{array}{c}\log \left[M(\mathrm{HI})_{\mathrm{obs}}\right] \\
\left(\log \left[M_{\odot}\right]\right) \\
\end{array}$ & $\begin{array}{c}\log \left[M(\mathrm{HI})_{\text {pred }}\right]^{a} \\
\left(\log \left[M_{\odot}\right]\right)\end{array}$ & $\begin{array}{c}\text { s.e.e. } \\
\left(\log \left[M_{\odot}\right]\right)\end{array}$ & Remarks \\
\hline 70 & 109.9 & 9.53 & 10.16 & 0.20 & triplet \\
\hline 72 & 170.0 & $<9.83$ & 10.33 & 0.27 & \\
\hline 73 & 181.4 & 10.84 & 10.11 & 0.22 & triplet \\
\hline 74 & 161.0 & $<10.08$ & 9.44 & 0.28 & no spiral members \\
\hline 76 & 137.0 & $<9.51$ & 10.05 & 0.20 & \\
\hline 78 & 122.0 & 10.40 & 10.24 & 0.22 & triplet \\
\hline 79 & 58.2 & 9.30 & 9.71 & 0.20 & \\
\hline 80 & 124.9 & 10.45 & 10.46 & 0.18 & \\
\hline 82 & 146.0 & $<9.69$ & 10.45 & 0.22 & \\
\hline 85 & 158.6 & $<9.72$ & 9.07 & 0.36 & no spiral members \\
\hline 87 & 116.7 & 9.59 & 10.47 & 0.18 & \\
\hline 88 & 80.7 & 10.28 & 10.55 & 0.17 & \\
\hline 89 & 119.6 & 10.45 & 10.49 & 0.14 & \\
\hline 91 & 95.7 & 10.32 & 10.56 & 0.17 & \\
\hline 92 & 86.4 & 10.02 & 10.51 & 0.17 & \\
\hline 93 & 67.4 & 9.56 & 10.36 & 0.24 & \\
\hline 94 & 168.4 & 10.22 & 10.06 & 0.17 & \\
\hline 95 & 159.8 & $>10.10$ & 10.31 & 0.24 & triplet \\
\hline 96 & 117.6 & $>10.20$ & 10.37 & 0.19 & \\
\hline 97 & 87.6 & 9.10 & 9.99 & 0.21 & \\
\hline 98 & 107.0 & 9.09 & 9.81 & 0.29 & no spiral members, triplet \\
\hline 99 & 116.7 & 9.21 & 10.11 & 0.26 & \\
\hline 100 & 71.5 & 9.74 & 10.24 & 0.18 & \\
\hline
\end{tabular}

a The derivation of the expected HI mass for the groups are discussed in Sect. 3.1.

are given for HCG 16, 95 and 96 because HI absorption strongly affects their spectra.

Out of the 70 galaxies (11 ellipticals) included in the VLA sample of the 16 HCGs, 50 galaxies are detected, and the HI emission is resolved for 34 galaxies in 12 groups. Good upper limits, ranging from $5 \times 10^{7}$ to $10^{8} M_{\odot}$, are obtained for the 9 undetected spiral galaxies. We measured the VLA HI flux associated with the main body of each galaxy, as well as that in gaseous tidal features, bridges and external clouds (Table 3). Separating HI fluxes for individual galaxies in HCG 18, 26, 49 and 54 was not possible because of confusion. Figure 3 shows the observed versus expected HI masses for individual galaxies, with the solid line representing a normal HI content. The HI in tidal features has not been included in the observed mass. For those cases where a tidal feature could be clearly associated to a particular member of a group, a dotted line connects the HI masses obtained with (open circle) and without (filled circle) the tidal feature. Arrows represent upper or lower limits (due to absorption features in the HI spectra). The mean HI deficiency of the 43 resolved spiral galaxies is $D e f_{\mathrm{HI}}=0.62 \pm 0.09$. This is significantly larger than the mean value for the groups, due in most cases to efficient gas stripping in these galaxies visible in the VLA maps. The most deficient galaxy is HCG 92b, undetected in HI, with $D e f_{\mathrm{HI}}>2.15$, which corresponds to $0.7 \%$ of the expected mass.

\section{Discussion}

\subsection{Possible systematic effects}

\subsubsection{Early type groups and the role of lenticulars}

Among the 72 groups in our sample, 8 do not contain any spiral member (HCG 8, 42, 46, 59, 62, 74, 85 and 98) hence the determination of the HI deficiency is highly uncertain. These groups lie mostly in the left part of Fig. 1 (large open circles), and when excluded from the calculation, the $\mathrm{HI}$ deficiency rises marginally to $D e f_{\mathrm{HI}}=0.35 \pm 0.06$. For the rest of the paper these 8 groups will be excluded from the calculations.

Since the expected HI content for S0 galaxies is generally uncertain, we have also calculated the decrease in expected HI mass if one assumes them to be devoid of HI (dashed lines in Fig. 1). In spite of the large number of lenticular galaxies in the sample $(22 \%)$, the change is only significant for a few groups, and the assumption leads to a global deficiency of $D e f_{\mathrm{HI}}=0.18 \pm 0.06$. On the other hand the early type population excess that exists in HCGs (Hickson et al. 1988) could suggest that some of them are stripped spirals (see e.g. Sulentic 2000). Hence for all groups with S0 members we have calculated the increase in predicted mass assuming that all were originally (conservatively) Sbs (thick lines in Fig. 1). Again this effect 
Table 3. HI masses for HCG galaxies.

\begin{tabular}{|c|c|c|c|}
\hline Galaxy $^{\mathrm{a}}$ & $\begin{array}{l}\log \left[M(\mathrm{HI})_{\text {obs }}\right] \\
\quad\left(\log \left[M_{\odot}\right]\right)\end{array}$ & $\begin{array}{c}\log \left[M(\mathrm{HI})_{\mathrm{pred}}\right]^{\mathrm{b}} \\
\left(\log \left[M_{\odot}\right]\right)\end{array}$ & $\begin{array}{c}\text { s.e.e. } \\
\left(\log \left[M_{\odot}\right]\right)\end{array}$ \\
\hline $2 \mathrm{a}$ & 10.18 & 10.07 & 0.30 \\
\hline $2 \mathrm{~b}$ & 9.19 & 9.73 & 0.30 \\
\hline $2 \mathrm{c}$ & 9.55 & 9.63 & 0.26 \\
\hline $2 \mathrm{~T}$ & 8.97 & & \\
\hline $16 \mathrm{a}$ & 9.07 & 9.79 & 0.38 \\
\hline $16 \mathrm{~b}$ & 8.90 & 9.66 & 0.38 \\
\hline $16 \mathrm{c}$ & 9.48 & 10.10 & 0.30 \\
\hline $16 \mathrm{~d}$ & $>9.65$ & 9.99 & 0.30 \\
\hline $16-3^{\mathrm{c}}$ & 9.64 & 9.57 & 0.38 \\
\hline $16-6^{\mathrm{c}}$ & 8.89 & 8.92 & 0.36 \\
\hline $16 \mathrm{~T}$ & 10.06 & & \\
\hline $23 a$ & 8.92 & 9.49 & 0.38 \\
\hline $23 \mathrm{~b}$ & 9.70 & 9.63 & 0.26 \\
\hline $23 \mathrm{c}$ & 8.36 & 8.95 & 0.63 \\
\hline $23 \mathrm{~d}$ & 9.15 & 9.27 & 0.30 \\
\hline $31 \mathrm{a}$ & 9.46 & 9.54 & 0.30 \\
\hline $31 b$ & 9.28 & 9.71 & 0.30 \\
\hline $31 \mathrm{c}$ & 9.20 & 10.32 & 0.30 \\
\hline $31 \mathrm{~g}$ & 9.28 & 9.54 & 0.30 \\
\hline $31 q$ & 8.95 & 9.07 & 0.30 \\
\hline $31 \mathrm{~T}$ & 10.12 & & \\
\hline $33 \mathrm{c}$ & 9.94 & 9.48 & 0.30 \\
\hline $40 \mathrm{~b}$ & $<8.22$ & 9.37 & 0.36 \\
\hline $40 \mathrm{c}$ & 8.96 & 9.66 & 0.29 \\
\hline $40 \mathrm{~d}$ & 8.52 & 9.63 & 0.38 \\
\hline $40 \mathrm{e}$ & $<8.22$ & 9.22 & 0.26 \\
\hline $44 a$ & 8.61 & 9.51 & 0.38 \\
\hline $44 \mathrm{c}$ & 8.41 & 9.40 & 0.26 \\
\hline $44 d$ & 8.92 & 9.33 & 0.30 \\
\hline $44 \mathrm{e}$ & 8.26 & 8.50 & 0.26 \\
\hline $49 \mathrm{~T}$ & 8.99 & & \\
\hline $79 b$ & $<7.72$ & 9.33 & 0.36 \\
\hline $79 \mathrm{c}$ & $<7.72$ & 9.09 & 0.36 \\
\hline $79 \mathrm{~d}$ & 9.14 & 9.23 & 0.30 \\
\hline $79 \mathrm{~T}$ & 8.40 & & \\
\hline $88 \mathrm{a}$ & 9.12 & 10.04 & 0.36 \\
\hline $88 \mathrm{~b}$ & 9.51 & 10.03 & 0.36 \\
\hline $88 \mathrm{c}$ & 10.01 & 9.90 & 0.26 \\
\hline $88 \mathrm{~d}$ & 9.58 & 9.74 & 0.26 \\
\hline $88 \mathrm{~T}$ & 8.46 & & \\
\hline $92 \mathrm{~b}$ & $<8.03$ & 10.18 & 0.29 \\
\hline $92 \mathrm{c}$ & $<8.03$ & 10.08 & 0.26 \\
\hline $92 \mathrm{~d}$ & $<8.03$ & 9.59 & 0.36 \\
\hline $92 \mathrm{f}$ & $<8.03$ & 9.03 & 0.38 \\
\hline $92 \mathrm{~T}$ & 9.98 & & \\
\hline $95 \mathrm{c}$ & $>9.41$ & 10.20 & 0.30 \\
\hline $95 \mathrm{~d}$ & $<8.70$ & 9.69 & 0.26 \\
\hline $95 \mathrm{~T}$ & 9.35 & & \\
\hline $96 \mathrm{a}$ & $>9.91$ & 10.21 & 0.26 \\
\hline $96 \mathrm{c}$ & 9.13 & 9.64 & 0.26 \\
\hline $96 \mathrm{~d}$ & 8.99 & 9.51 & 0.30 \\
\hline $96 \mathrm{~T}$ & 9.73 & & \\
\hline
\end{tabular}

a A $\mathrm{T}$ indicates the HI mass in tidal features external to the galaxies, as tails and bridges.

b HI mass expected for the group obtained as explained in Sect. 3.1.

c Notation from De Carvalho et al. (1997).

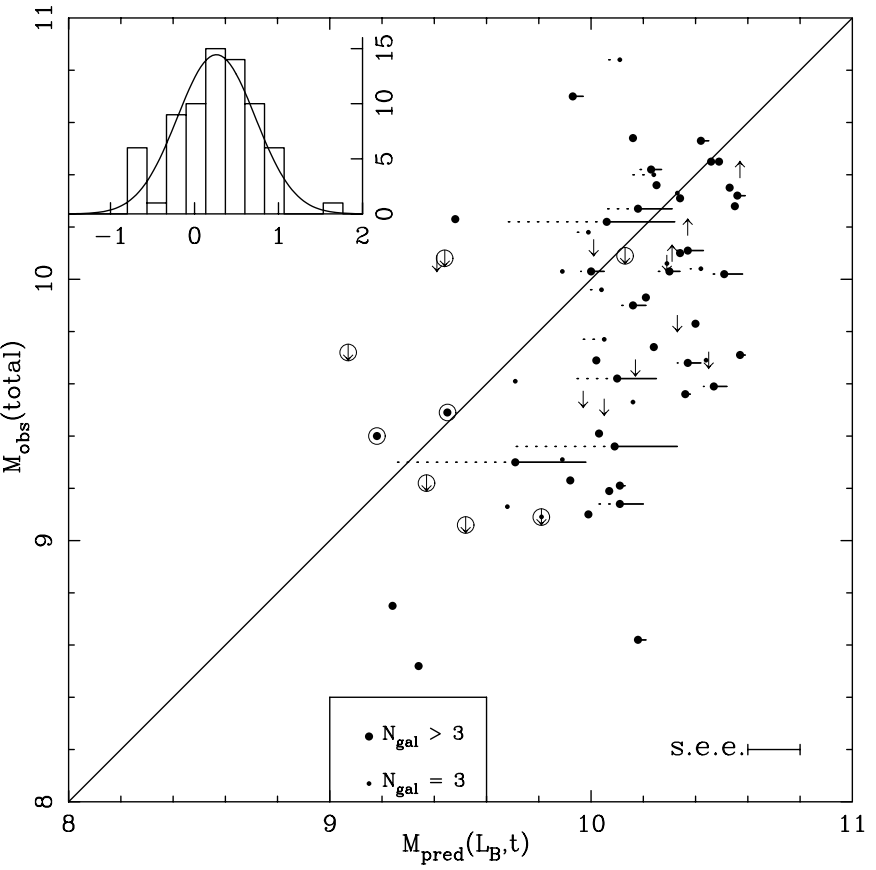

Fig. 1. Comparison of the total observed HI mass in each HGC with HI single-dish data with the expected one obtained as described in Sect. 3.1. Triplets are marked with smaller symbols. Dashed lines indicate the decrease in expected HI mass if one assumes lenticulars to be devoid of HI. Solid lines correspond to the increase in the predicted mass of groups containing lenticular galaxies assuming that all were originally Sbs. Arrows represent upper and lower limits. Big open circles indicate groups with no spiral members. The segment in the lower right indicates the mean value of the standard estimate of the error (s.e.e.). The solid line represents a normal HI content. An histogram of the HI deficiency for the 72 groups of the sample is shown in the inset in the upper left of the figure.

would be important just for a few groups, and the mean deficiency would be $D e f_{\mathrm{HI}}=0.40 \pm 0.06$.

\subsubsection{Beam size and $\mathrm{HI}$ extent}

We have considered whether the HI deficiency might be due to a small HI beam diameter $\left(D_{\text {beam }}\right)$. If true, the HI deficiency should be larger for groups with larger ratios of the HI extent $\left(D_{\mathrm{HI}}\right)$ with respect to the beam size. Since $D_{\mathrm{HI}}$ cannot be derived from the existing single-dish data, we have measured it for the groups mapped at the VLA and compared with their optical extent $\left(D_{\text {opt }}\right)$ which is obviously known for the whole sample. $D_{\mathrm{HI}}$ has been determined at the lowest common HI column density that can be measured $\left(7.7 \times 10^{19}\right.$ at cm$\left.^{-2}\right)$ (Table 1$)$ and the largest HI extent $\left(\sim 20^{\prime}\right.$ for HCG 16$)$ is well within the VLA primary beam of $30^{\prime}$. We find that $D_{\mathrm{opt}}=0.6-1.7 \times D_{\mathrm{HI}}$ and all mapped groups have within the optical diameter $\left(D_{\mathrm{opt}}\right.$, given in Hickson 1982 and revised here in order to exclude interlopers) at least $85 \%$ of the detected HI mass which is within the intrinsic errors in the determination of $M(\mathrm{HI})_{\text {pred }}$. Hence, in those cases where the single-dish beam is equal to or larger than the optical extent of the 


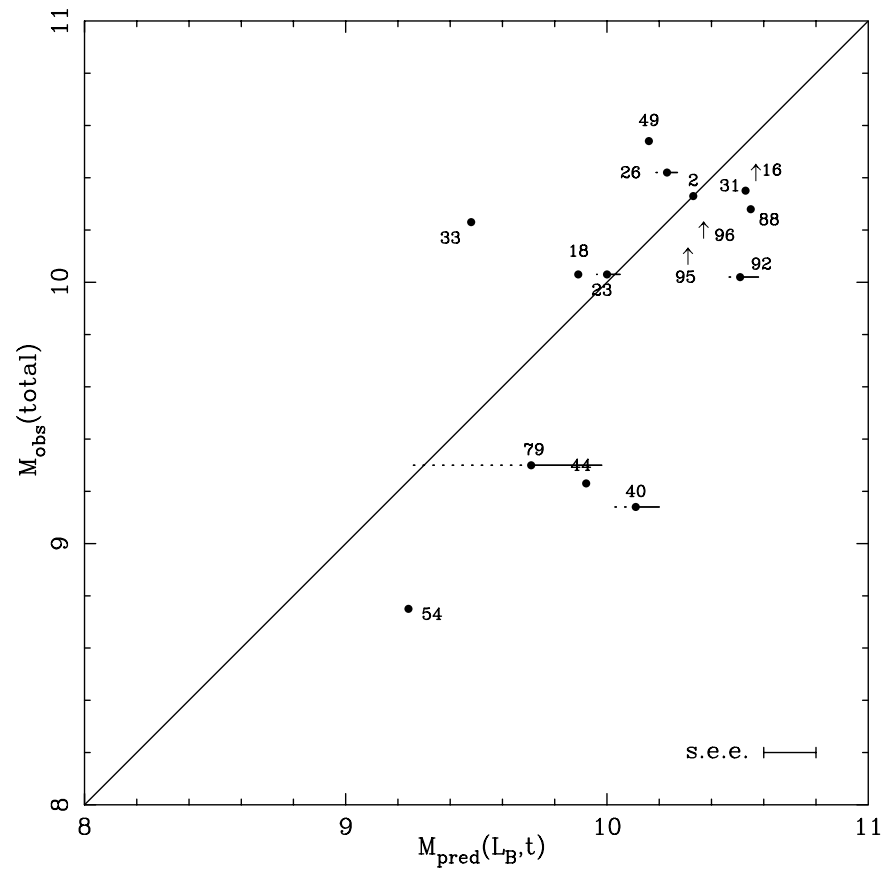

Fig. 2. Comparison of the total observed HI mass in each Hickson compact group observed with the VLA with the expected one obtained as described in Sect. 3.1. Symbols have the same meaning as in Fig. 1.

group no significant effect would be expected in the observed HI deficiency. This condition is met by 70 out of the 72 groups observed with single dish, and the remaining ones, HCG 10 and 21 are not classified as HI deficient. This rough calculation suggests that the beam size did not have a significant effect on the reported HI deficiency.

The reasoning above assumes that the total HI extent is given by the size measured in the VLA maps. Alternatively, the HI deficiency could be due to extended but undetected diffuse HI emission either within the $30^{\prime}$ VLA primary beam, or extending even farther away. If the "empty" area of the VLA beam is filled with part of the missing HI and the extension of the detected HI fills only a small percentage of the single-dish beam, then the single-dish flux should be larger than the VLA flux. The premises are met for the HI deficient groups but no difference is found between the single-dish and VLA fluxes within the typical sensitivities of the single-dish measurements, $\sim 10^{8} M_{\odot}$, while $\sim 10^{9}-10^{10} M_{\odot}$ are typically missing in the HI deficient groups (Table 2). This result is obtained for only a few groups, although including cases of extreme HI deficiency. Hence this indicates that the existence of significant undetected amounts of diffuse cold gas does not seem a general or at least the only explanation for the HI deficiency.

\subsubsection{Morphological misclassification}

Estimation of the HI mass expected for a given galaxy depends on its morphology. Galaxies in compact groups

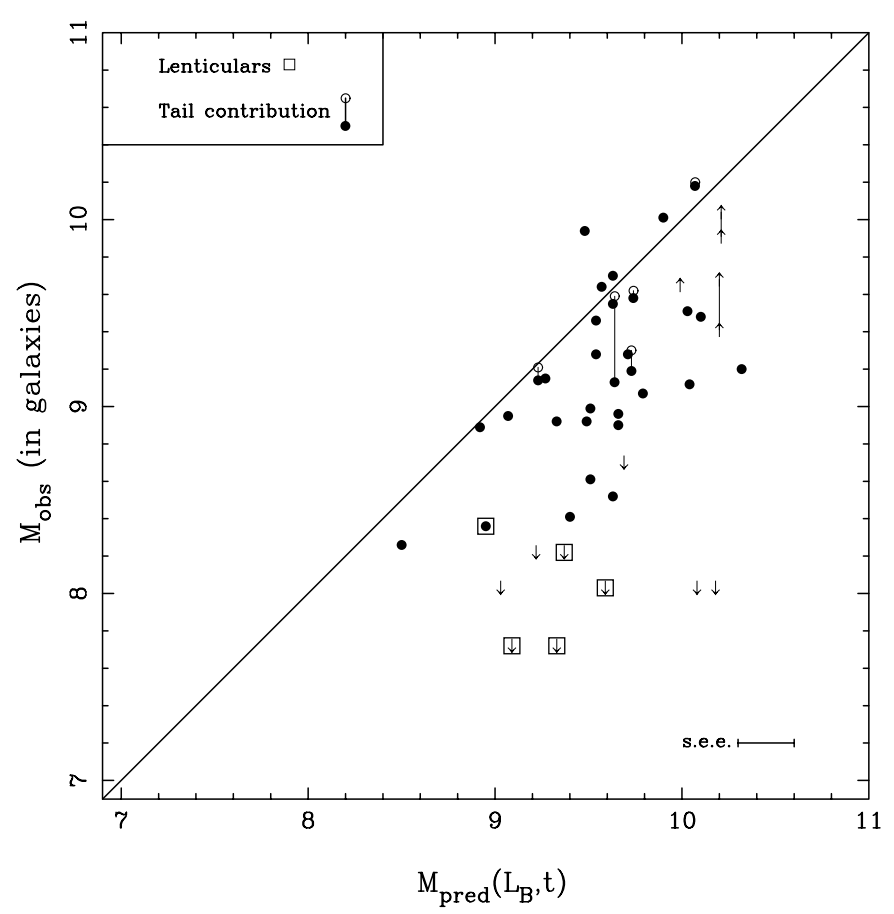

Fig. 3. Comparison of the measured HI mass in the galaxies of the groups observed with the VLA with the expected one for their optical luminosities and morphological types, obtained as described in Sect. 3.1. Lenticular galaxies are marked with squares. The vertical segments connect the masses obtained excluding (filled circle) and including (open circle) the tidal feature. Arrows represent upper or lower limits.

show distortions that can complicate a reliable determination of their morphological type $t$ (as presented in Hickson et al. 1989, e.g. $t(\mathrm{Sa})=3, t(\mathrm{Sb})=5)$, hence a source of scatter that can mask real trends. For galaxies earlier than approximately $t=8(\mathrm{Scd})$ errors larger than $\Delta t=2$ would be infrequent, while for later types errors of the order of $\Delta t=4$ might occur. The morphology of $85 \%$ of the galaxies in the studied groups are earlier than $t=8$, ranging from E to Sc (Fig. 4), so that large errors are not expected. The most probable effect produced by tidal interactions would be disk stripping, hence favoring errors of the order of $\Delta t=-2$. We have quantified this effect by comparing the total $\mathrm{HI}$ masses expected for groups where the morphological types of its $N$ members are $t_{(i=1, N)}$ and those obtained using $t_{(i=1, N)}-2$ (Fig. 5). Therefore, in the improbable case that all galaxies have been misclassified systematically by $\Delta t=-2, D e f_{\mathrm{HI}}$ would be increased by 0.18 . For a random error of the order of \pm 2 the spread in the HI deficiency would be increased by a similar amount, but the fraction of deficient groups would be still significant and not the result of a wrong determination of morphology.

\subsubsection{Triplets and false groups in the HCG sample}

According to Hickson classification (Hickson 1982; Hickson et al. 1992), a total of 15 triplets exist in the total sample, marked with smaller symbols in Fig. 1, or 14 in 


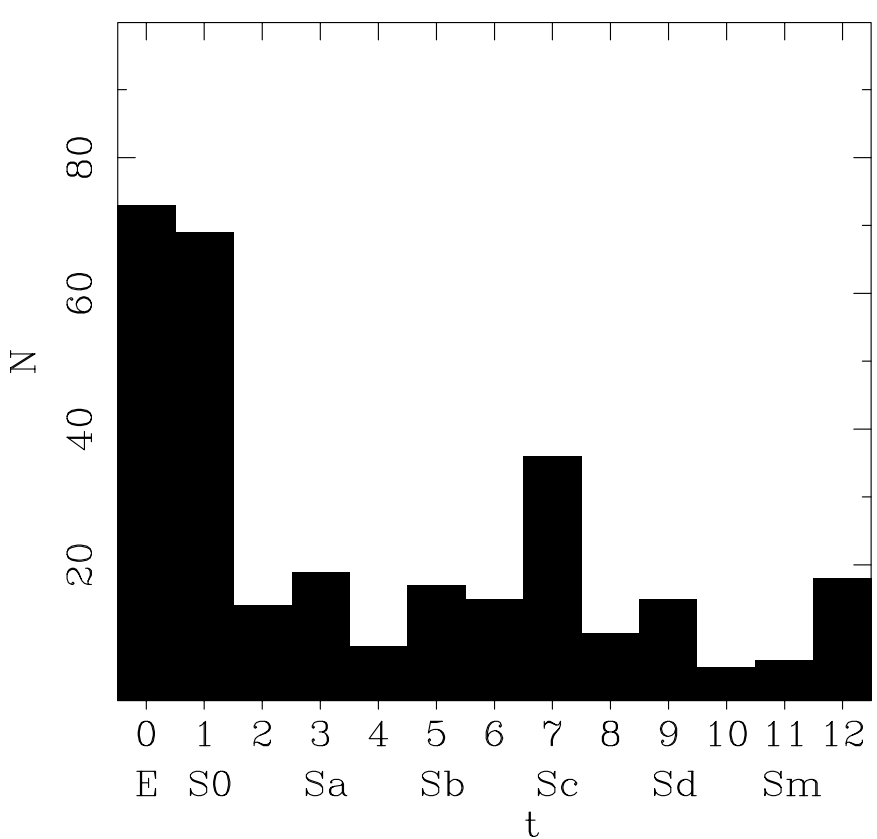

Fig. 4. A histogram of the morphological types for the 309 galaxies included in the 72 groups with HI single-dish data. The numerical assignment of the Hubble types are also given under the $x$-axis.

our reduced sample of 64 groups (when groups with no spirals are removed). We analyze them separately since their physical reality as dynamical entities is not clearly established (Thomas \& Couchamn 1992). Some of them might be HCGs in formation, in a scenario as the one suggested by Governato et al. (1996), where groups form and are maintained against collapse by accretion of new members. They might also be the result of some merging in CGs, but no sign of that is evident from the existing observations. The HI deficiency of the triple systems is not significant, $D e f_{\mathrm{HI}}=0.19 \pm 0.10$, which can be understood from the above considerations, as either not real systems or unevolved systems. One of these triplets, HCG 2, has been mapped by us at the VLA, and the only signs of interactions found are incipient HI tails arising from 2 of its members. When these systems are excluded from the sample, the HI deficiency of the 50 groups with at least 4 members (and at least one spiral) increases marginally to $D e f_{\mathrm{HI}}=0.40 \pm 0.07$.

A few cases are found in our sample that might be false groups, in the sense that instead of being separate galaxies they might well be knots of an irregular galaxy, or at most 2 galaxies. This is the case of HCG 18, which is probably a single knotty galaxy (see e.g. Williams \& Van Gorkom 1988; Plana et al. 2000). HCG 54 is also composed of small optical knots ( $\sim 0.6 \mathrm{kpc}$ diameter), embedded in a single cloud with a diameter of $12 \mathrm{kpc}$ with a long tail about $20 \mathrm{kpc}$ long. We are currently investigating the existence of some neighbor galaxy that might have produced the tidal tail. When removed from the sample the deficiency does not change significantly.

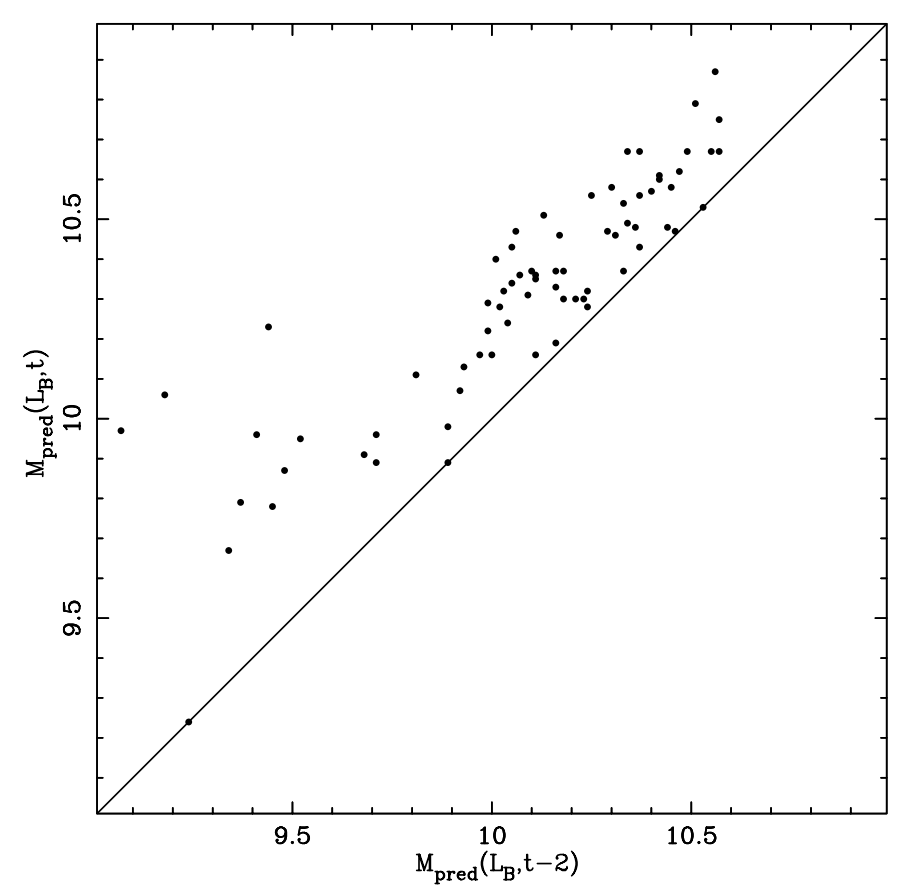

Fig. 5. Comparison of the total HI masses expected for groups whose morphological types are $t_{(i=1, N)}$ versus those obtained using $t_{(i=1, N)}-2$. This plot demonstrates the effect of misidentifying the Hubble type by $\Delta t=2$.

\subsection{HI deficiency and group properties}

The most HI deficient groups among those mapped with the VLA are HCG 40, 44 and 92, while the existence of two lenticular galaxies in HCG 79 makes its deficiency uncertain. We find that the HI deficiency in them is not due to a few highly anemic objects, but shared by all of their members. Although this result is obtained based on a small set of groups, it suggests that the cause of HI deficiency should be associated with a group property.

In this section we explore possible parameters that can be the origin of the HI deficiency found in HCGs. We will exclude from all calculations groups with no spirals, triplets and false groups, so that the final sample is composed of 48 groups, with $D e f_{\mathrm{HI}}=0.40 \pm 0.07$. For the purpose of the statistical calculations performed in this section the sample has been divided between normal HI groups and HI deficient groups according to the definition given in Sect. 3.1. HI excess has not been considered since only 3 groups would be, in this case, and derived quantities will not have statistical significance. The rest divides equally between normal and deficient groups.

\subsubsection{Morphology of the galaxies}

The presence of a 1st-ranked elliptical galaxy, acting as a deep potential well, has been suggested in the literature as the cause of the HI deficiency through consumption or ionization of the gas (Vílchez \& Iglesias-Páramo 1998; Sulentic \& de Mello Rabaca 1993). From our data the difference in HI deficiency between groups with a 1st-ranked 
Table 4. Mean physical parameters for the HCGs.

\begin{tabular}{lccc}
\hline Parameter $^{\mathrm{a}}$ & HI normal & HI deficient & Difference in $\sigma$ \\
\hline Fraction of ellipticals & 0.14 & 0.25 & 2.2 \\
Fraction of E+S0 & 0.30 & 0.44 & 2.0 \\
$C o m p /\left(L_{\left.\mathrm{B} ~ \mathrm{Mpc}^{-2}\right)}\right.$ & 390 & 888 & 2.5 \\
$N$ & 4.6 & 4.9 & 1.1 \\
$D_{\mathrm{G}}(\mathrm{kpc})$ & 136.8 & 107.5 & 1.5 \\
$R_{25}(\mathrm{kpc})$ & 11.9 & 12.7 & 0.6 \\
$R_{\mathrm{P}}(\mathrm{kpc})$ & 1.7 & 1.6 & 0.7 \\
$\log \sigma_{\mathrm{v}}\left(\mathrm{km} \mathrm{s}{ }^{-1}\right)$ & 2.14 & 2.31 & 2.0 \\
$\Delta \rho$ & 316 & 557 & 1.0 \\
$n_{\text {corr }}$ & 1.1 & 2.2 & 1.3 \\
$D e f_{\mathrm{H}_{2}}$ & -0.21 & 0.25 & 3.2 \\
X-ray detected & $4 / 22$ & $10 / 22$ & \\
\hline
\end{tabular}

a The listed parameters are defined in Sect. 4.2. All of them are mean values of group parameters calculated on the basis of the final sample of 48 HCGs, except for $D e f_{\mathrm{H}_{2}}$ and for the $\mathrm{X}$-ray detection rate. $D e f_{\mathrm{H}_{2}}$ is calculated for the 32 galaxies with both $\mathrm{HI}$ and $\mathrm{CO}$ data. The X-ray detection rate is given for the 44 groups with HI data observed in X-ray by Ponman et al. (1996).

elliptical and with a 1st-ranked spiral, or even when no elliptical galaxies exist, is not significant. Therefore a bright elliptical does not appear to play an important role in the HI deficiency, although it cannot be excluded that this mechanism is at work in some particular cases. Only a marginal $(2 \sigma)$ trend is found for HI deficient groups to be richer in early type galaxies (Table 4 ).

\subsubsection{Group compactness, velocity dispersion, isolation and related parameters}

Do the most compact groups show also the largest HI deficiencies? Since the densest groups should include the most dynamically evolved, compactness is a parameter to investigate in this study. We compared compactness of normal and HI deficient groups (Comp, calculated as the total $L_{\mathrm{B}}$ of accordant galaxies divided by the physical surface area of the group) and found weak $(2.5 \sigma)$ evidence for the HI deficient groups to show higher surface density (Table 4). No correlation is found between the number of galaxies in each group $(N)$, group diameter $\left(D_{\mathrm{G}}\right)$, galaxy sizes $\left(R_{25}\right)$, and median projected separation of the galaxies $\left(R_{\mathrm{p}}\right)$ and the HI deficiency. HI deficient groups have a marginal $(2 \sigma)$ trend to have larger velocity dispersions $\left(\sigma_{\mathrm{v}}\right)$ than normal groups.

HCGs were defined with an isolation criterion (Hickson 1982); however, new data have permitted refinements in the characterization of their environment, showing a variety of situations. Measuring on the red POSS plates, Sulentic (1987) performed counts of galaxies density enhancement within $0.5^{\circ}$ of HCGs and within one magnitude of the faintest galaxy in the group $(\Delta \rho)$.
Rood \& Williams (1989) performed similar counts within 10 radii of the group, and apparent magnitude in the range of the group members, correcting for the estimated number of field galaxies $\left(n_{\text {corr }}\right)$. We find no difference in the degree of isolation of normal and HI deficient groups (Table 4).

\subsubsection{Interstellar medium}

Gravitational torque during encounters can produce higher density conditions due to accretion of $\mathrm{HI}$ toward the center, inducing conversion of atomic into molecular gas (García-Barreto et al. 1994; Menon 1995 and references therein). CO data exist for 79 among 202 spirals in 44 of the groups with single-dish HI data (Verdes-Montenegro et al. 1998; Leon et al. 1998), and no CO excess has been found. Instead, $20 \%$ of the groups are in fact $\mathrm{CO}$ poor. For the 32 galaxies with both $\mathrm{CO}$ and $\mathrm{HI}$ data, we have compared $\mathrm{HI}$ and $\mathrm{H}_{2}$ deficiency $\left(\right.$ De $f_{\mathrm{H}_{2}}=$ $\log \left[M\left(\mathrm{H}_{2}\right)_{\text {pred }}\right]-\log \left[M\left(\mathrm{H}_{2}\right)_{\mathrm{obs}}\right]$, with $M\left(\mathrm{H}_{2}\right)_{\text {pred }}$ obtained from Perea et al. 1997). We find that galaxies with a larger amount of stripped HI show a trend to have a depressed CO content ( $3 \sigma$, Table 4). Quilis et al. (2000) suggested that HI stripping would break the balance between disruption of molecular clouds by star formation and condensation of new molecular complexes, so producing $\mathrm{H}_{2}$ deficiency and consequently depressed star formation activity. A general comparison between star formation activity (e.g. star formation rate or far-infrared emission) and $\mathrm{HI}$ content has not been possible due to the small number of galaxies common to both samples.

Sixty-four of the 72 HCGs with HI data have been observed in X-ray by Ponman et al. (1996), 44 of them belong to our final sample of 48 (22 normal in HI and 22 deficient), and 3 more are only composed of only early type galaxies. Among them only 14 have associated (diffuse) X-ray emission according to Ponman et al. (1996). This leaves us little cases for a statistical study of X-ray luminosities. However the detection rate is more than double for $\mathrm{HI}$ deficient groups (Table 4), and we have checked that this is not due to larger exposure times or closer distances. This suggests that HI deficient groups are more luminous at $\mathrm{X}$-ray wavelengths. Not much information can be obtained from VLA mapped groups: only HCG 33, HCG 16 and HCG 92 have been detected in X-ray and no maps exist for HCG 33. Recent XMM images of HCG 16 (Turner et al. 2001) indicate that the X-ray emission is not diffuse but comes from the nuclei of the galaxies. This is consistent with the normal HI content of this group. In the case of HCG 92 the anticorrelation between HI and X-ray emission suggests that the missing atomic gas was heated in a shock after being removed from the individual galaxies (see Sect. 4.3). These evidences are not general enough to be applied to the whole sample.

The largest deficiency among the 72 groups with single-dish measurements is found in HCG 30, with De $f_{\mathrm{HI}}=1.56(97 \%)$. HCG 30 is composed of 4 galaxies 


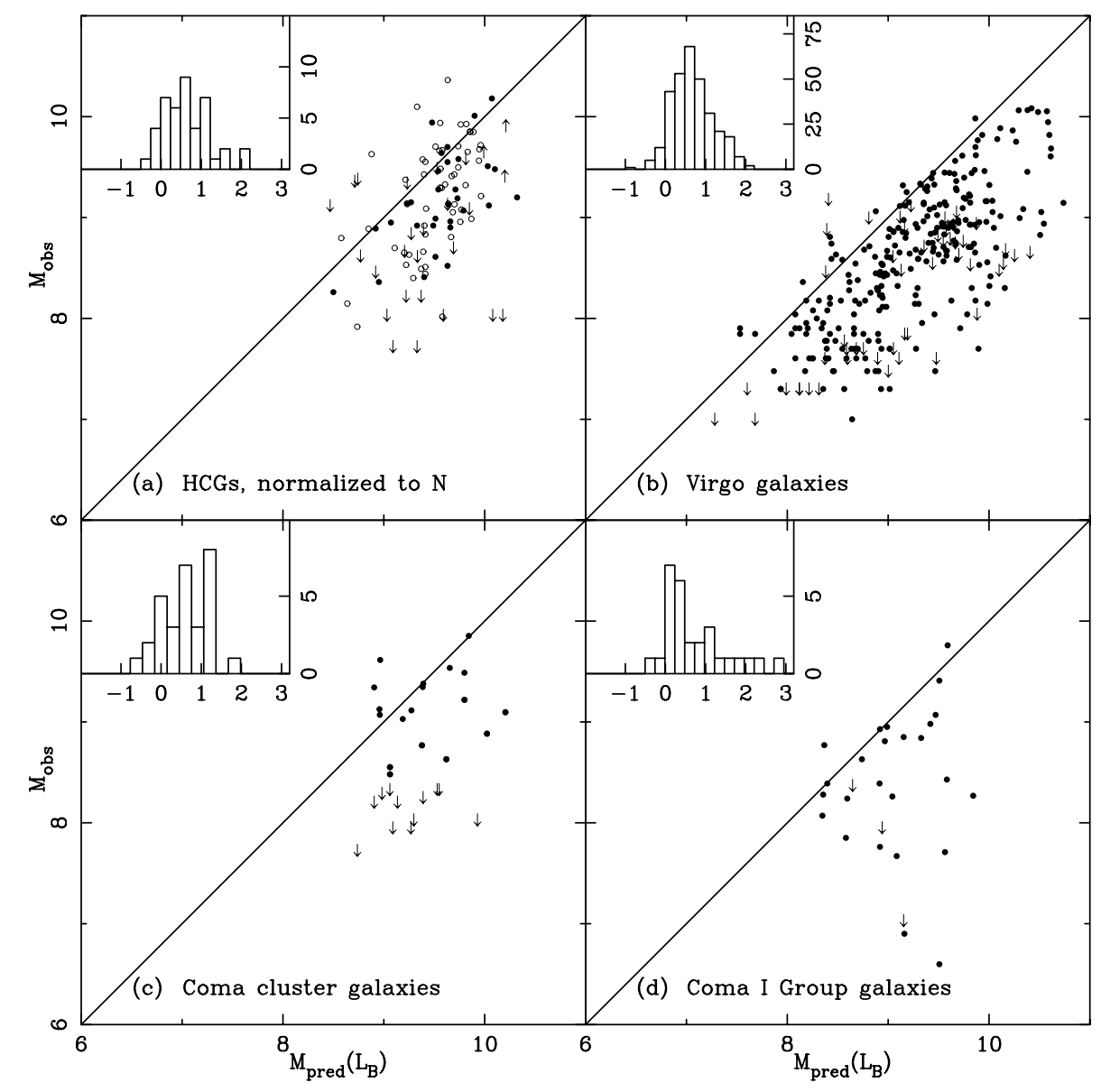

Fig. 6. Observed versus predicted HI mass for: a) HCGs as a whole (filled circles) where the total masses have been normalized to the number of galaxies in the group for comparison, and for the individual galaxies (open circles); b) Virgo galaxies (Huchtmeier and Richter 1989); c) Coma cluster galaxies (Bravo-Alfaro et al. 2000); d) Coma I group (García-Barreto et al. 1994). Histograms of the HI deficiency for each sample are shown in the insets of the upper left of each panel.

(3 spirals and 1 lenticular) with optical luminosities $L_{\mathrm{B}}=$ $7,5,3$ and $0.8 \times 10^{9} L_{\odot}$, but only $4 \times 10^{8} M_{\odot}$ of atomic gas is detected. We did not detect CO toward this group, with an upper limit for each galaxy of $9 \times 10^{8} M_{\odot}$, a factor of 10 lower than expected for the spirals, the same applying to the FIR luminosity $\left(3 \times 10^{9} L_{\odot}\right.$ observed versus $3 \times 10^{10} L_{\odot}$ expected for each spiral; Verdes-Montenegro et al. 1998). Little $\mathrm{H} \alpha$ emission is detected in the four galaxies of this group (Vílchez \& Iglesias-Páramo 1998), consistent with the low amount of gas associated with them. Finally, the density contrast with respect to the environment is among the lowest. In a radius of $500 \mathrm{kpc}$ four more galaxies with magnitudes in the range of the group members and velocity differences below $300 \mathrm{~km} \mathrm{~s}^{-1}$ are listed in NED.

The most extreme cases of HI deficient galaxies $\left(D e f_{\mathrm{HI}}>1\right)$ mapped at the VLA are HCG $92 \mathrm{~b}$ and c where no $\mathrm{HI}$ is detected above a $3 \sigma$ value of $0.8 \%$ of their expected content. These galaxies are strongly deficient in molecular gas, with $D e f_{\mathrm{H}_{2}}>1.2$ for HCG $92 \mathrm{~b}$ and $D e f_{\mathrm{H}_{2}}=1.0$ for HCG 92c (Yun et al. 1997). The FIR emission of these galaxies is not enhanced with respect to their optical luminosity, indicating that disk star formation was not triggered by the interactions.

\subsubsection{Comparison with other dense environments}

We have compared the degree of HI deficiency in HCGs with galaxies in Virgo and Coma clusters as well as in the Coma I group. In Fig. 6 we compare the observed and expected values of $\mathrm{HI}$ mass for these environments. Histograms of the HI deficiency are also shown. The HI data for individual galaxies of HCGs are shown in Fig. 6a as filled circles, and the mean value for the groups, obtained after normalizing to the number of members, as open circles. In Fig. 6b we plot the data for 313 galaxies in Virgo cluster within 10.5 of M87 (Huchtmeier \& Richter 1989). Data for Coma cluster galaxies are plotted in Fig. 6c (Bravo-Alfaro et al. 2000) and for Coma I group in Fig. 6d (García-Barreto et al. 1994). In the 3 comparison samples the largest deficiencies are reached close to the center of the cluster/group and with values similar to those found in HCGs, in spite of the different characteristics of each environment. Deficiencies are larger for Coma I group galaxies and is probably due to a better constraint on the upper limits due to its closer distance. Clusters have larger number of members and velocity dispersions than groups, and ram pressure stripping 
is the standard explanation there for HI deficiency. Coma I cloud is composed of 32 galaxies, half of them HI deficient, with radial velocity dispersion similar to compact groups $\left(\sigma=190 \mathrm{~km} \mathrm{~s}^{-1}\right)$. García-Barreto et al. (1994) argue that the corresponding pressure of a hypothetical intragroup medium could strip gas from the outer part of the disks. Recent numerical simulations such as by Abadi et al. (1999) have found that ram pressure stripping is not an efficient mechanism in a group and poor cluster environment, and another mechanism is likely needed to account for the intragroup medium.

The question on how such intragroup medium can be generated or mimicked in the compact group environment maybe related with the fact that the number of crossings are much larger than in clusters, where most galaxies would instead suffer fewer but more violent passages through the cluster center. A second alternative, where gas is heated by shock induced by an intruder galaxy was discussed in Sect. 4.2.3 in the context of X-ray emission.

\subsection{An evolutionary sequence from the HI distribution}

The groups imaged with the VLA show a variety of HI distributions, with $70 \%$ of the spiral galaxies perturbed in $\mathrm{HI}$, and their morphology and kinematics, as well as HI content, may be interpreted as different stages of group evolution. There is no one-to-one correspondence between the presence of tidal features and atomic gas content. Not all deficient groups show HI tidal tails, neither are all groups with HI tidal features deficient in HI, e.g. HCG 16, 31, 92 and 96 show numerous tidal tails although HCG 92 is the only HI deficient.

The normal level of FIR emission found among the HCG galaxies (Verdes-Montenegro et al. 1997) is not consistent with the usual scenario of tidally induced gas inflow seen among interacting galaxies and the contemporaneous, enhanced starburst activity. Although the inflow of gas and conversion into stars during the past episodes of interactions cannot be ruled out, efficient tidal removal of the outer HI disks and the inhibition of inflow (see Sect. 4.2.3) offers a simpler explanation. Once the HI is tidally removed from the individual galaxies, the dynamic and energetic compact group environment may be highly inducive to dispersal or ionization of the gas and producing the observed HI deficiency. As discussed by Hibbard et al. (2000), HI tidal tails are dynamically fragile and may be easily ionized, thereby possibly explaining why some HI deficient groups do not show tidal tails. The expected emission measure for ionized HI tidal tails is of order $1 \mathrm{~cm}^{-6} \mathrm{pc}$ or less, which is detectable only by the most sensitive observations. This leads us to propose a scheme for an evolutionary sequence, shown in Fig. 7 with a sample group in each class. HCG 95 is not considered here since only half of the single-dish HI flux is detected in the VLA maps, and found to be located in the galaxies (Huchtmeier et al. 2000).

In Phase 1 the HI distribution and kinematics are relatively unperturbed, and more than $\sim 90 \%$ of the HI mass is located in the disks of the galaxies with the remaining gas found in incipient tidal tails. Examples of this are HCG 88 (top of the tree in Fig. 7) together with HCG 23 and 33. HCG 88 has a high density of galaxies $\left(160\right.$ galaxies $/ \mathrm{Mpc}^{2}$ ) and high degree of isolation (only two galaxies, 4 magnitudes fainter than galaxy d, are found in a 700 pc-side square centered on the group; De Carvalho et al. 1997), and is especially interesting since this quartet of spirals, aligned in projection, has been considered a good candidate for a projected filament (Hernquist et al. 1995). However the properties of this group, in particular velocity dispersion $\left(\sigma_{\mathrm{v}}=27 \mathrm{~km} \mathrm{~s}^{-1}\right)$ and crossing time $\left(H_{0} t_{\mathrm{c}}=8.70\right)$, are quite different from those predicted by the simulations performed by Hernquist et al. (1995; see their Table 1). Therefore we think that HCG 88 is a good example of a physically dense group at a very early stage of interaction.

In Phase 2, groups still retain a significant amount of $\mathrm{HI}$ in the disks of the spirals but 30 to $60 \%$ of the total HI mass forms tidal features. The HI morphology in these groups can be understood as an extrapolation of interactions in pairs of galaxies, where tails and bridges are often seen. Examples are HCG 96, 16 and 31, ordered from lower to higher percentage of gas in tidal features. In the figure we show HCG 16 as an example where the intragroup atomic gas composes a grid of bridges and tails.

In Phase 3 a most if not all of the HI have been stripped from the disks of the galaxies and are either found in tails, produced in multiple tidal interactions, or not even detected. Examples of this are HCG 40, 44 and 92. HCG 92 is a specially striking case since all the observed $\mathrm{HI}(35 \%$ of the expected content) has been stripped out from the galaxies and formed several intragalactic clouds and tidal tails (Williams et al. 2001). Abundant heated and/or ionized gas is observed: X-ray emission from shocked gas (Pietsch et al. 1997) anticorrelates with the HI, and intense bursts of star formation external to the galaxies have been reported by several authors (e.g. Moles et al. 1997; Plana et al. 1999; Xu et al. 1999; Gallagher et al. 2000). The most extreme case is HCG 40, where HI is only detected in a fraction of the disks of two of its spiral members, and no HI external to the galaxies is detected, so that $10^{10} M_{\odot}(90 \%$ of the expected HI) are missing. We think that the HI was stripped and then suffered more easily a phase change, although this requires further confirmation since no X-ray measurement exists for this group.

The least common Phase (named 3b in Fig. 7) involves groups where the HI gas forms a large cloud containing all galaxies, and with a single velocity gradient. The total HI spectrum has a characteristic single peaked shape. This appears to be a rare situation, since among the 72 groups observed with single dish (excluding HCG 18 and HCG 54 as probable false groups, see Sect. 4.1.4) only HCG 26 (Williams \& Van Gorkom 1995) and 49 show this kind of spectrum. HCG 26 is dominated by a large edge-on spiral whose HI is difficult to differentiate from the whole cloud. HCG 49 is the best defined and most impressive example of this phase, shown in the right branch of Fig. 7. The HI 


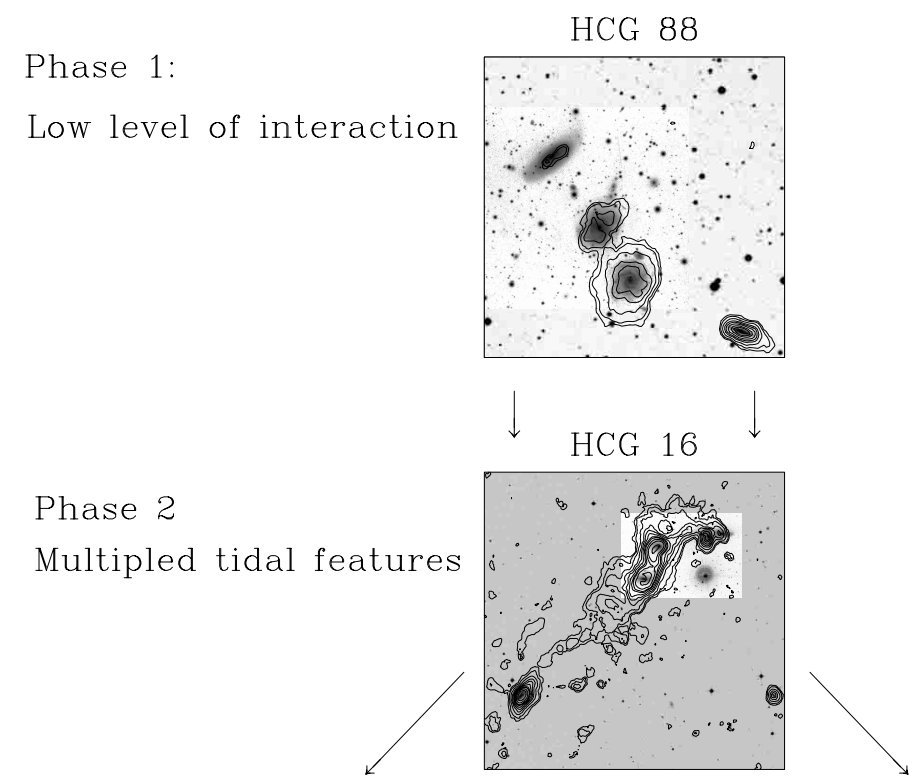

Phase 3a: No HI in the galaxies

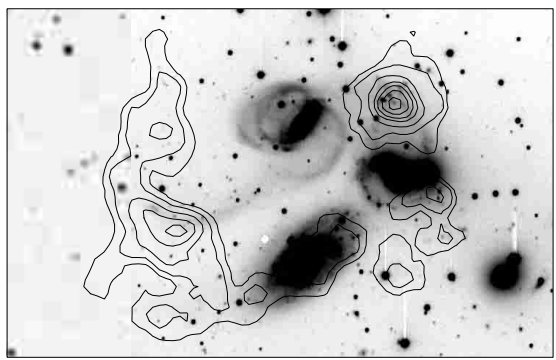

HCG 92

Phase 3b: HI in an envelope
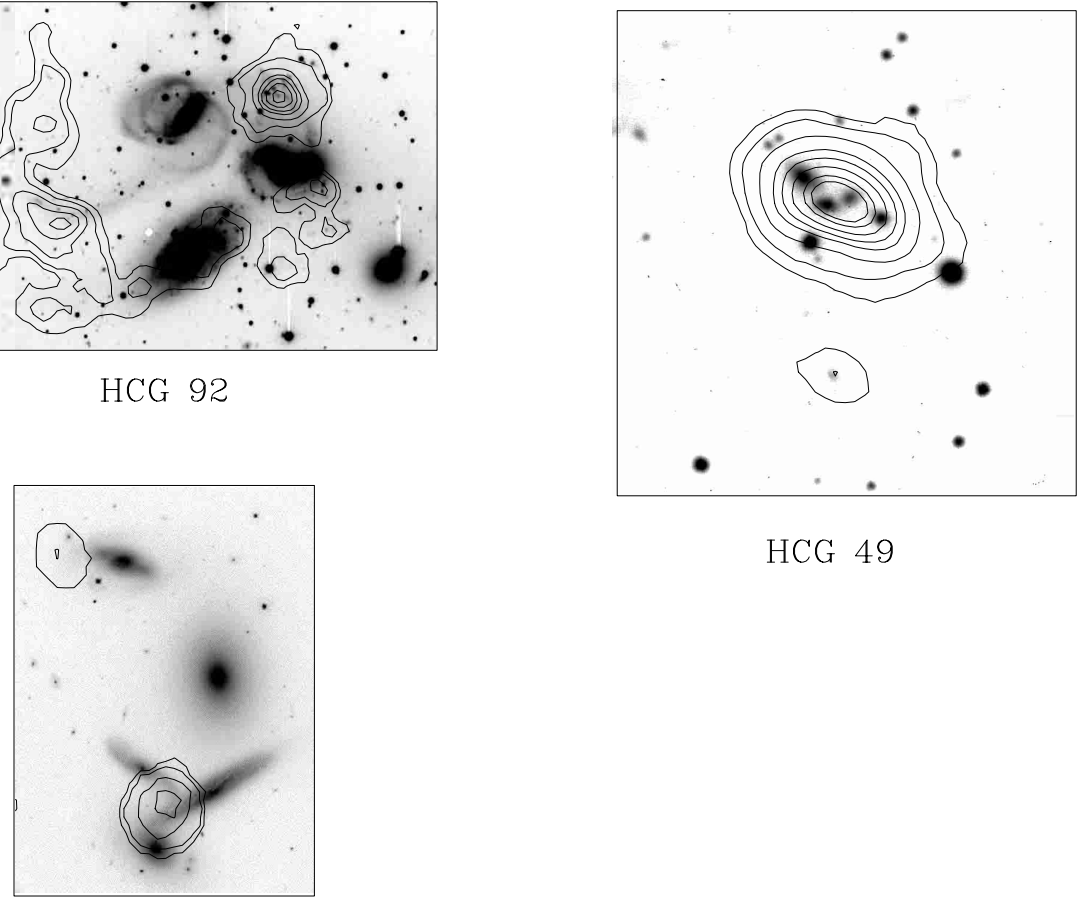

HCG 49

HCG 40

Fig. 7. A proposed evolutionary scenario for HCGs (see Sect. 4.3). The optical image for HCG 88 is a superposition of a POSS image and a CCD $V$ image obtained with NOT in La Palma. In the case of HCG 16 an $R$ image (kindly given by Iglesias-Páramo) is superposed on a POSS image. The $R$ optical image shown for HCG 92 was taken by Sulentic at the $3.5 \mathrm{~m}$ telescope of Calar Alto. A $B$ image is shown for HCG 49, obtained at the $1.5 \mathrm{~m}$ telescope of OSN (Granada). The image of HCG 40 was taken with Subaru (Subaru Telescope is operated by the National Astronomical Observatory of Japan) in 1999 (http:www.naoj.org) and is a combination of $J$ and $K^{\prime}$ frames. The shown levels are $N(\mathrm{HI})=10,20,40,65,85,110,140,160,200,250,350,450,570 \times$ $10^{19}$ at $\mathrm{cm}^{-2}$.

cloud has an elliptical shape with a size of $95 \times 65 \mathrm{kpc}$, and a velocity gradient of $\sim 250 \mathrm{~km} \mathrm{~s}^{-1}$, containing 4 well differentiated galaxies with a velocity dispersion as low as $34 \mathrm{~km} \mathrm{~s}^{-1}$ and a total optical diameter of $35 \mathrm{kpc}$. The morphology and kinematics of this cloud (Fig. 8a) is not likely the result of smoothed emission from multiple tidal tails, like those found in HCG 31 (Fig. 8b). Smoothing the HCG 31 datacube to the same physical resolution as in HCG 49 (Fig. 8c) gives a similar overall morphology but different kinematics, since no continuous velocity gradient is obtained for HCG 31 with a lower resolution. The galaxies of this group are similar in luminosity and type to M33, for which extended HI envelopes are not unusual (Huchtmeier 1973). More extended atomic gas would be 


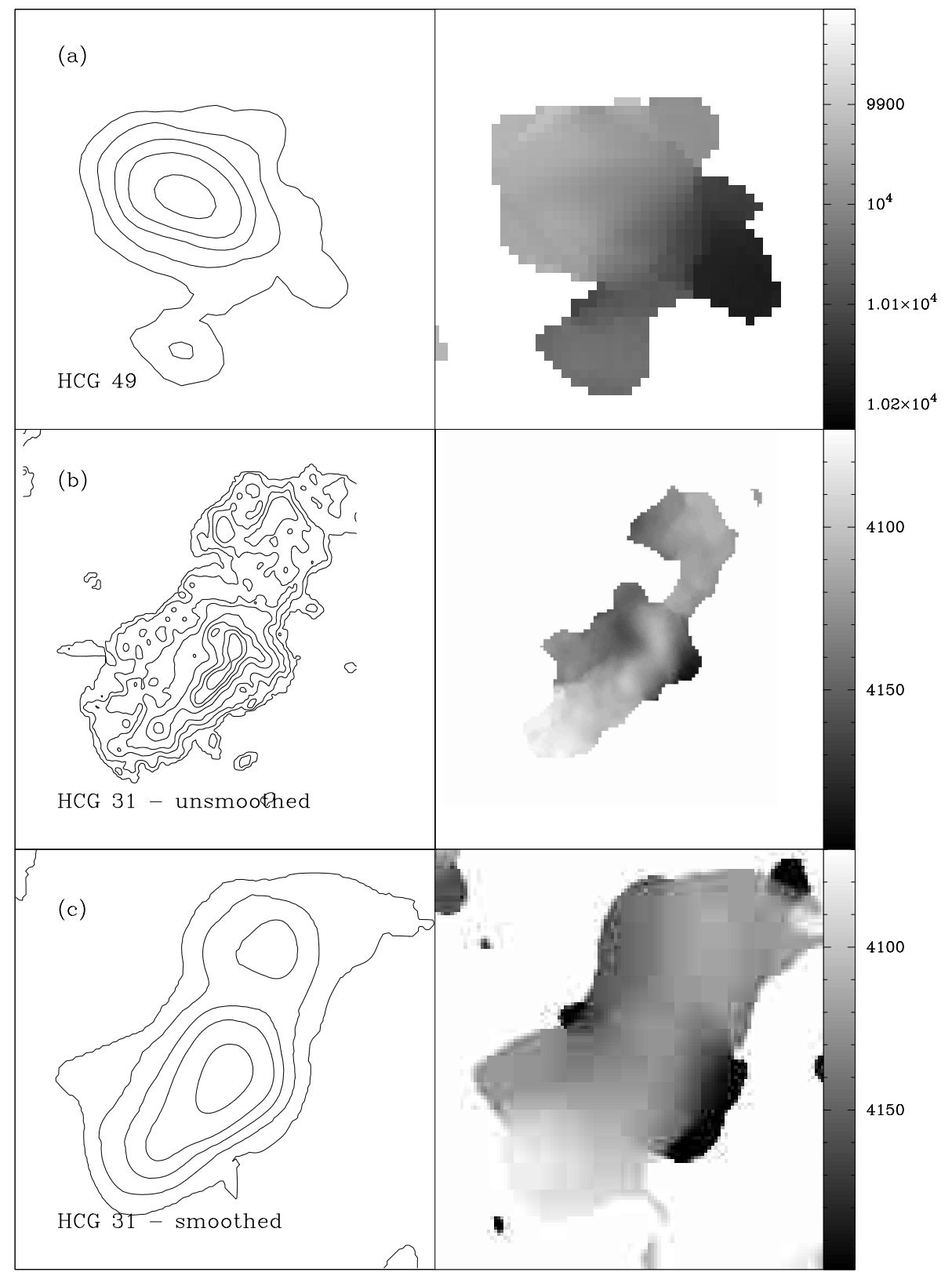

Fig. 8. A test of resolution effects. a) HI integrated emission (left) and velocity field (right) of HCG 49. b) The same as in a) for HCG 31. c) The same as in b) smoothed to a beam with the same physical size as in HCG $49\left(63^{\prime \prime} \times 52^{\prime \prime}\right)$. The shown levels are $N(\mathrm{HI})=4,18,40,72,143,206,252,295 \times 10^{19}$ at $^{-2}$.

more easily detached from the individual members to form a common HI cloud (A. Bosma, priv. comm.). Hence evolution from Phase 2 to $3 \mathrm{a}$ or $3 \mathrm{~b}$ would depend on the size of the galaxies. Small optical disks with respect to the HI extent may favor the formation of a single cloud. We are currently performing optical imaging and spectroscopy of this group in order to better characterize the dynamnics of the group.

The case of HCG 79 (Seyfert's sextet) is considered here separately due to the uncertainty in its classification. This group contains a giant elliptical with a prominent dust lane as well as FIR and radiocontinuum emission, two lenticulars and a single spiral galaxy, from which an $\mathrm{HI}$ and 2 optical tails are emerging. However the signs of dynamical evolution are stronger in the optical, with an extended luminous halo (Sulentic \& Lorre 1983; Nishiura et al. 2000). The detected HI tail might have been produced via interaction of the spiral member with this halo.

\section{Conclusions}

A combined analysis of single-dish data for 72 HCGs and high resolution mapping for 16 shows:

- The full sample of 72 groups with HI single-dish data has a mean HI deficiency of Def $f_{\mathrm{HI}}=0.36 \pm 0.06$. When a more homogeneus sample is extracted, excluding triplets, false groups, and groups with no spiral members, the remaining 48 groups show a larger mean 
HI deficiency of $0.40 \pm 0.07$. These values depend only weakly on expected errors in the classification of the morphological types;

- The galaxies mapped with the VLA have a larger degree of deficiency, $D e f_{\mathrm{HI}}=0.62 \pm 0.09$ in agreement with the efficient gas stripping that takes place, evidenced by HI tails and bridges;

- We have investigated whether the deficiency could be due to HI missed from the beam, either due to a small beamsize relative to the group extent, or to the presence of diffuse undetected emission. If the HI extent observed in the VLA maps is reasonably representative of the whole sample, then the beam size did not have a relevant effect on the reported HI deficiency. We discard also the presence of significant amounts of extended cold gas that escaped the observations, from comparison between VLA and single-dish fluxes;

- A large set of group parameters is explored as the origin of the $\mathrm{HI}$ deficiency, including morphological types, size of the groups and galaxies, compactness, velocity dispersion, isolation and ISM. We find a weak $(2-2.5 \sigma)$ trend for the HI deficient groups to be early type rich, more compact, and show higher velocity dispersions. A slightly stronger correlation exists $(3 \sigma$ level) with the $\mathrm{CO}$ content: HI deficient groups have also depressed CO emission. The study of a larger and complete sample (as the one defined by Pradoni et al. 1994; see also Coziol et al. 2000) would be desirable to confirm these trends;

- The most extreme case of deficient group (HCG 30; $D e f_{\mathrm{HI}}=1.56$ or $97 \%$ of the expected HI missing) as well as deficient galaxies (HCG 92b and d; Def $f_{\mathrm{HI}}=$ $2.1-99 \%$ ) show also a depressed content of molecular gas and no FIR enhancement. Star formation seems therefore inhibited by intense HI stripping from the galactic disks that breaks the ISM equilibrium;

- The HI deficiency level is similar to the one found in the central galaxies of Coma and Virgo clusters, as well as Coma I group, although there the deficiencies seems to be related to the existence of a hot intragroup medium. The existence of a similar mechanism in groups is not clear. Among the 44 groups of our sample observed in X-ray, the detection rate is higher for the HI deficient groups, and this is not due to closer distances or longer exposure times. However the distribution of the X-ray emission is not clear. HCG 16 shows only nuclear X-ray emission while HCG 92 shows heated gas cooling at X-ray wavelengths, with the hot gas anticorrelated with the neutral atomic gas;

- The mapped groups show a variety of HI distributions, with $70 \%$ of the spiral galaxies perturbed in HI. These results are combined in a possible evolutionary scenario. Least evolved groups are found with a low level of interaction. Later, multiple tidal tails form, a stage that can be understood as an extrapolation of pair interactions. Once the gas is removed from the galaxies it can be more easily heated or destroyed, thereby producing $\mathrm{HI}$ deficient groups. In a few cases, the HI evolves to form a single cloud containing the whole group, which might be related to the smaller size of the galaxies.

The general process producing $\mathrm{HI}$ deficiency in a large number of HCGs is yet to be clarified. Our results suggest that gas heating maybe playing a role, but more sensitive X-ray fluxes and maps for a larger set of groups with available HI data would substantially help to improve our understanding.

Acknowledgements. We acknowledge interesting discussions with J. Sulentic, P. T. P. Ho, R. Sancisi, E. Athanassoula and A. Bosma. LV-M, AO and JP are partially supported by DGI (Spain) Grant AYA2000-1564 and Junta de Andalucía (Spain).

\section{References}

Abadi, M. G., Moore, B., \& Bower, R. G. 1999, MNRAS, 308, 947

Athanassoula, E., Makino, J., \& Bosma, A. 1997, MNRAS, 286,825

Bravo-Alfaro, H., Cayatte, V., van Gorkom, J. H., \& Balkowski, C. 2000 , AJ, 119,580

De Carvalho, R. R., Ribeiro, A. L. B., Capelato, H. V., \& Zepf, S. E. 1997, ApJS, 110, 1

Coziol, R., Iovino, A., \& de Carvalho, R. R. 2000, AJ, 120, 47 Diaferio, A., Geller, M. J., \& Ramella, M. 1994, AJ, 107, 868

Gallagher, S. C., Hunsberger, S. D., Charlton, J. C., \& Zaritsky, D. 2000, in Massive Stellar Clusters, Proceedings of the international workshop held in Strasbourg, France, November 8-11, 1999, ed. A. Lançon, \& C. Boily, ASP Conf. Ser., 247

García-Barreto, J. A., Downes, D. A., \& Huchtmeier, W. K. 1994, A\&A, 288, 705

Governato, F., Tozzi, P., \& Cavaliere, A. 1996, ApJ, 458, 18

Haynes, M. P., \& Giovanelli, R. 1984, AJ, 89, 758

Hernquist, L., Katz, N., \& Weinberg, D. H. 1995, ApJ, 442, 57

Hibbard, J. E., Vacca, W. D., \& Yun, M. S. 2000, AJ, 119, 1130

Hibbard, J. E., \& Van Gorkom, J. H. 1997, AJ, 111

Hickson, P. 1982, ApJ, 255, 382

Hickson, P., Kindl, E., \& Auman, J. R. 1988, ApJ, 331, 64

Hickson, P., Kindl, E., \& Auman, J. R. 1989, ApJS, 70, 687

Hickson, P., Mendes de Oliveira, C., Huchra, J. P., \& Palumbo, G. 1992, ApJ, 399, 353

Huchtmeier, W. K. 1973, A\&A, 22, 91

Huchtmeier, W. K. 1997, A\&A, 325, 473

Huchtmeier, W. K., \& Richter, O.-G. 1989, A\&A, 210, 1

Huchtmeier, W. K., Verdes-Montenegro, L., Yun, M. S., Del Olmo, A., \& Perea, J. 2000, Small Galaxy Groups: IAU Colloq., 174, ed. M. J. Valtonen, \& C. Flynn, ASP Conf. Ser., 209 (Astronomical Society of the Pacific), 154

Leon, S., Combes, F., \& Menon, T. K. 1998, A\&A, 330, 37

Menon, T. K. 1995, MNRAS 274, 845

Moles, M., del Olmo, A., Perea, J., et al. 1994, A\&A, 285, 444

Moles, M., Sulentic, J. W., Márquez, I., et al. 1997, ApJ, 485, L69

Nishiura, S., Murayama, T., Shimada, M., et al. 2000, AJ, 120, 2355 
Perea, J., del Olmo, A., Verdes-Montenegro, L., \& Yun, M. S. 1997, ApJ, 490, 166

Perea, J., del Olmo, A., Verdes-Montenegro, L., et al. 2000, Small Galaxy Groups: IAU Colloq., 174, ed. M. J. Valtonen, \& C. Flynn, ASP Conf. Ser., 209 (Astronomical Society of the Pacific), 377

Pietsch, W., Trinchieri, G., Arp, H., \& Sulentic, J. W. 1997, A\&A, 322, 89

Plana, H., Amram, P., Mendes de Oliveira, C., \& Balkowski, C. 2000, AJ, 120,621

Plana, H., Mendes de Oliveira, C., \& Amram, P. 1999, ApJ, $516, \mathrm{~L} 69$

Ponman, T. J., Bourner, P. D. J., Ebeling, H., \& Bohringer, H. 1996, MNRAS, 283, 690

Prandoni, I., Iovino, A., \& MacGillvray, H. T. 1994, AJ, 107, 1235

Price, R. M., Babic, B., \& Jones, K. 2000, Small Galaxy Groups: IAU Colloq., 174, ed. M. J. Valtonen, \& C. Flynn, ASP Conf. Ser., 209 (Astronomical Society of the Pacific), 163

Quilis, V., Moore, B., \& Bower, R. 2000, Science, 288, 1617

Rood, H. J., \& Williams, B. A. 1989, ApJ, 339, 772

Shostak, G. S., Sullivan III, W. T., \& Allen, R. J. 1984, A\&A, 139,15

Sulentic, J. W. 1987, ApJ, 322, 605

Sulentic 2000, Small Galaxy Groups: IAU Colloq., 174, ed. M. J. Valtonen, \& C. Flynn, ASP Conf. Ser., 209 (Astronomical Society of the Pacific), 227

Sulentic, J. W., \& Lorre, J. J. 1983, A\&A, 120, 36

Sulentic, J. W., \& Rabaca, C. R. 1994, ApJ, 429, 531

Sulentic, J. W., \& de Mello Rabaca, D. F. 1993, ApJ, 410, 520

Turner, M. J. L., et al. 2001, A\&A, 365, L11

Thomas, P. A., \& Couchman, H. M. P. 1992, MNRAS, 257, 11
Verdes-Montenegro, L., Del Olmo, A., Perea, J., et al. 1997, A\&A, 321, 409

Verdes-Montenegro, L., Yun, M. S., Perea, J., Del Olmo, A., \& Ho, P. T. P. 1998, ApJ, 497, 89

Verdes-Montenegro, L., Yun, M. S., \& Williams, B. A. 2000a, Small Galaxy Groups: IAU Colloq., 174, ed. M. J. Valtonen, \& C. Flynn, ASP Conf. Ser., 209 (Astronomical Society of the Pacific), 167

Verdes-Montenegro, L., Yun, M. S., Williams, B. A., et al. 2000b, Gas and Galaxy Evolution: A Conference in Honor of the 20th Anniversary of the VLA (Socorro, New Mexico, USA)

Vílchez, J. M., \& Iglesias-Páramo, J. 1998, ApJ, 506, L101

Williams, B. A., McMahon, P. M., \& Van Gorkom, J. H. 1991, AJ, 101, 1957

Williams, B. A., \& Rood, H. J. 1987, ApJS, 63, 265

Williams, B. A., \& Van Gorkom, J. 1988, AJ, 95, 352

Williams, B. A., \& Van Gorkom, J. H. 1995, in Groups of galaxies, ASP Conf. Ser., 70, 77

Williams, B. A., Van Gorkom, J. H., Yun, M. S., \& VerdesMontenegro, L. 1997, in IAU Conf. Galaxy Interactions at Low and High Redshift (Kyoto, Japan)

Williams, B. A., Yun, M. S., \& Verdes-Montenegro, L. 1999, AAA, 194, 19.05

Williams, B. A., Yun, M. S., \& Verdes-Montenegro, L. 2001, in preparation

Xu, C., Sulentic, J. W., \& Tuffs, R. 1999, ApJ, 512, 178

Yun, M. S., Ho, P. T. P., \& Lo, K. Y. 1994, Nature, 372, 530

Yun, M. S., Verdes-Montenegro, L., Del Olmo, A., \& Perea, J. 1997, ApJ, 475, L21

Zepf, S. E., Whitmore, B. C., \& Levison, H. F. 1991, ApJ, 383, 524 